\title{
The Carcinogenic Liver Fluke Opisthorchis viverrini is a Reservoir for Species of Helicobacter
}

\author{
Raksawan Deenonpoe ${ }^{1,2}$, Chariya Chomvarin ${ }^{3}$, Chawalit Pairojkul' ${ }^{2}$, Yaowalux \\ Chamgramol $^{2}$, Alex Loukas ${ }^{4}$, Paul J Brindley ${ }^{5}$, Banchob Sripa ${ }^{1,2 *}$
}

\begin{abstract}
There has been a strong, positive correlation between opisthorchiasis-associated cholangiocarcinoma and infection with Helicobacter. Here a rodent model of human infection with Opisthorchis viverrini was utilized to further investigate relationships of apparent co-infections with $O$. viverrini and $H$.pylori. A total of 150 hamsters were assigned to five groups: $i$ ) Control hamsters not infected with $O$. viverrini; ii) $O$. viverrini-infected hamsters; iii) non- $O$. viverrini infected hamsters treated with antibiotics $(\mathrm{ABx})$; $i$ v) $O$. viverrini-infected hamsters treated with $\mathrm{ABx}$; and v) $O$. viverrini-infected hamsters treated both with $\mathrm{ABx}$ and praziquantel (PZQ). Stomach, gallbladder, liver, colonic tissue, colorectal feces and $O$. viverrini worms were collected and the presence of species of Helicobacter determined by PCR-based approaches. In addition, $O$. viverrini worms were cultured in vitro with and without $\mathrm{ABX}$ for four weeks, after which the presence of Helicobacter spp. was determined. In situ localization of $\boldsymbol{H}$.pylori and Helicobacter-like species was performed using a combination of histochemistry and immunohistochemistry. The prevalence of $H$. pylori infection in $O$. viverrini-infected hamsters was significantly higher than that of $O$. viverrini-uninfected hamsters $(p \leq 0.001)$. Interestingly, $O$. viverrini-infected hamsters treated with $A B x$ and PZQ (to remove the flukes) had a significantly lower frequency of $H$.pylori than either $O$. viverriniinfected hamsters treated only with $\mathrm{ABx}$ or $O$. viverrini-infected hamsters, respectively (p $\leq 0.001)$. Quantitative RT-PCR strongly confirmed the correlation between intensity $H$. pylori infection and the presence of liver fluke infection. In vitro, $H$. pylori could be detected in the $O$. viverrini worms cultured with ABx over four weeks. In situ localization revealed $\boldsymbol{H}$. pylori and other Helicobacter-like bacteria in worm gut. The findings indicate that the liver fluke $O$. viverrini in the biliary tree of the hamsters harbors $H$. pylori and Helicobacter-like bacteria. Accordingly, the association between $\boldsymbol{O}$. viverrini and $\boldsymbol{H}$. pylori may be an obligatory mutualism.
\end{abstract}

Keywords: Opisthorchis viverrini - Helicobacter - H. pylori - reservoir host - hamster

Asian Pac J Cancer Prev, 16 (5), 1751-1758

\section{Introduction}

Cholangiocarcinoma (CCA) is highly prevalent in Asian countries, particularly in Thailand, China, Taiwan and Korea (Shin et al., 2010). The principal risk factor for $\mathrm{CCA}$ in this region is infection with the human liver flukes, Opisthorchis viverrini and Clonorchis sinensis, which are Group 1 carcinogens as classified by the World Health Organization (Sripa et al., 2007; 2012; Bouvard et al., 2009; IARC, 2012). Thailand has the highest incidence of CCA worldwide due to its robust association with opisthorchiasis (Sripa and Pairojkul, 2008; Shin et al., 2010). However, several other risk factors for this cancer have been documented including primary sclerosing cholangitis (see Rizvi and Gores, 2013), inflammatory bowel disease (Huai et al., 2014), metabolic syndromes (Wu et al., 2012), hepatitis virus (Matsumoto et al., 2014) and infection with Helicobacter spp. (Zhou et al., 2013). The latter has attracted increasing research interest in recent years (Mateos-Munoz et al., 2013; Murphy et al., 2014).

Helicobacter pylori infection was the first bacterial infection known to be a causative factor of gastrointestinal diseases including gastric adenocarcinoma (Bouvard et al., 2009). Its virulence factors such as cagA and vacA are involved in the pathogenesis of the diseases (Cid et al., 2013; Hatakeyama, 2014). Other Helicobacter species, specifically $H$. hepaticus and $H$. bilis also are implicated in hepatobiliary disease (Zhou et al., 2013; MateosMuñoz et al., 2013; Murphy et al., 2014). We observed an association between $H$. pylori and hepatolithiasis or CCA in people in Northeast Thailand, a region endemic for opisthorchiasis (Boonyanugomol et al., 2012b; 2012c). Molecular mechanisms integral to $H$. pylori

${ }^{1}$ WHO Collaborating Centre for Research and Control of Opisthorchiasis (Southeast Asian Liver Fluke Disease), Tropical Disease Research Laboratory, ${ }^{2}$ Department of Pathology, ${ }^{3}$ Department of Microbiology, Faculty of Medicine, Khon Kaen University, Khon Kaen, Thailand, ${ }^{4}$ Australian Institute of Tropical Health \& Medicine, James Cook University, Cairns, QLD, Australia, ${ }^{5}$ Department of Microbiology, Immunology \& Tropical Medicine, and Research Center for Neglected Diseases of Poverty, School of Medicine \& Health Sciences, The George Washington University, Washington, DC, USA *For correspondence: banchob@kku.ac.th 
induced hepatobiliary diseases have also been reported (Boonyanugomol et al. 2011; 2012a). However, the underlying mechanisms by which species of Helicobacter associate with opisthorchiasis remain unclear. Here we investigated the prospective inter-relationship between infections with Helicobacter spp. and liver flukes in a rodent model of this foodborne liver fluke infection.

\section{Materials and Methods}

\section{Animals}

Female Syrian hamsters, Mesocricetus auratus, about 8 weeks of age were housed in conventional conditions, fed a commercial diet and given water ad libitum. Rodent husbandry conformed to the ethical guidelines of the Animal Laboratory Center, Faculty of Medicine, Khon Kaen University; these studies were approved by the Animal Ethics Committee of Khon Kaen University; approval number AEKKU \# 100/2555. Laboratory infection of hamsters with $O$. viverrini was accomplished by oral administration of 50 metacercariae, as described (Lvova et al., 2012). Infected hamsters were maintained up to three months after the infection; control, non-infected hamsters were housed in identical conditions.

\section{Experimental design}

The hamsters $(n=150)$ were assigned to five groups of 30 rodents each (Table 1): group 1 - uninfected controls $(n=30)$; group 2 - hamsters treated with antibiotics $(A B x)$ (clarithromycin $7.5 \mathrm{mg} / \mathrm{kg} \mathrm{BID+} \mathrm{metronidazole} \mathrm{20mg/}$ $\mathrm{kg}$ BID + amoxicillin $20 \mathrm{mg} / \mathrm{kg}$ BID), omeprazole 0.7

Table 1. Treatment Groups of Hamsters Infected with the Liver Fluke Opisthorchis viverrini

\begin{tabular}{lcccc}
\hline Group & $\begin{array}{c}\text { Number of } \\
\text { hamsters }\end{array}$ & $\begin{array}{c}\text { Infection with } \\
\text { O. viverrini }\end{array}$ & $\mathrm{ABx}$ & PZQ \\
\hline 1 & 30 & - & - & - \\
2 & 30 & + & - & - \\
3 & 30 & - & + & - \\
4 & 30 & + & + & - \\
5 & 30 & + & + & + \\
\hline
\end{tabular}

$\mathrm{mg} / \mathrm{kg}$ BID and sucralfate $300 \mathrm{mg} / \mathrm{kg}$ ) twice a day for 14 days, as described (Khoshnegah et al., 2011); group 3 - O. viverrini infected hamsters; group 4 - O viverriniinfected hamsters treated with $\mathrm{ABx}$ for 14 days; group 5 - O. viverrini-infected hamsters treated with $\mathrm{ABx}$ for 14 days and then treated with praziquantel (PZQ) (Biltricide, Bayer, Pittsburgh, PA) suspended in $2 \%$ Cremophor EI (Sigma, St. Louis, MO) at $400 \mathrm{mg} / \mathrm{kg}$ body weight twice, with an interval of two weeks between treatments aiming to eradicate the parasites. Hamsters were euthanized, after which colorectal feces, stomach, gallbladder, liver and worms were collected from each group for DNA extraction. Some worms were cultured for up to four weeks in RPMI 1640 medium supplemented with penicillin and streptomycin. Samples of livers from selected hamsters were fixed in $10 \%$ buffered formalin for histochemistry and immunohistochemistry.

\section{DNA extraction}

One gram of stool sample was used for DNA extraction using a QIAamp ${ }^{\circledR}$ DNA Stool Mini Kit (Qiagen, Germany). The worms, liver, stomach, gallbladder and colon were incubated with lysis buffer (10 mM Tris- $\mathrm{HCl}$ pH 8.5, 10 mM EDTA, $100 \mathrm{mM} \mathrm{NaCl}, 0.5 \%$ SDS), homogenized with tissue grinder, and digested with proteinase $\mathrm{K}$ at $55^{\circ} \mathrm{C}$ overnight. Thereafter, DNAs were recovered using precipitation with ethanol.

\section{Molecular detection of Helicobacter species by PCR}

Fifty ng DNA from each stool was used in the PCR assay. The reaction mixture consisted of 1x GoTaq ${ }^{\circledR}$ Colorless Master Mix (Promega, USA) containing 0.2 mM $\mathrm{dNTP}, 1.5 \mathrm{mM} \mathrm{MgCl} 2$, primers at $0.2 \mathrm{mM}$ and $1.25 \mathrm{U}$ of Taq DNA polymerase. Primer sequences for the detection of Helicobacter spp.(16srRNA), H.pylori (ureA), H.bilis, $H$. hepaticus are provided in Table 2. PCR was performed in a GeneAmp ${ }^{\circledR}$ PCR system 9700 (Applied Biosystems, Life Technologies) thermocycler. Amplified products were sized by electrophoresis through $1.0 \%$ agarose, stained with ethidium bromide and visualized under a UV illuminator.

Table 2. Primer Sequences and PCR Conditions for the Detection of Species of Helicobacter

\begin{tabular}{|c|c|c|c|c|}
\hline Genes & Primer sequences & PCR conditions & Product size (bp) & References \\
\hline \multirow[t]{2}{*}{$\begin{array}{l}\text { 16Sr RNA } \\
\text { (nested PCR) }\end{array}$} & $\begin{array}{l}\text { OF-ATTAGTGGCGCACGGGTGAGTAA } \\
\text { OR-TTTAGCATCCCGACTTAAGGC }\end{array}$ & $\begin{array}{l}94 \mathrm{oC} 30 \mathrm{sec}, 55 \mathrm{oC} 30 \mathrm{sec} \text {, } \\
72 \mathrm{oC} 1.5 \mathrm{~min}(35 \mathrm{cycles}) \\
94 \mathrm{oC} 30 \mathrm{sec}, 60 \mathrm{oC} 30 \mathrm{sec} \text {, }\end{array}$ & 1,300 & \multirow[t]{2}{*}{$\begin{array}{l}\text { Pellicano et al. } \\
(2004)\end{array}$} \\
\hline & $\begin{array}{l}\text { IF-GAACCTTACCTAGGCTTGACATTG } \\
\text { IR-GGTGAGTACAAGACCCGGGAA }\end{array}$ & $72 \mathrm{oC} 30 \mathrm{sec}(35$ cycle & 480 & \\
\hline \multirow[t]{2}{*}{$\begin{array}{l}\text { ureA } \\
\text { (nested PCR) }\end{array}$} & $\begin{array}{l}\text { OF-GCTAATGGTAAATTAGTTCCTGG } \\
\text { OR-CTCCTTAATTGTTTTTACATAGTTG }\end{array}$ & $\begin{array}{l}94 \mathrm{oC} 30 \mathrm{sec}, 62 \mathrm{oC} 30 \mathrm{sec}, \\
72 \mathrm{oC} 30 \mathrm{sec}(40 \text { cycles })\end{array}$ & 411 & \multirow[t]{2}{*}{$\begin{array}{l}\text { Pellicano et al. } \\
\text { (2004) }\end{array}$} \\
\hline & $\begin{array}{l}\text { IF-AGTTCCTGGTGAGTTGTTCTTAA } \\
\text { IR-AACCACGCTCTTTAGCTCTGTC }\end{array}$ & $\begin{array}{l}94 \mathrm{oC} 30 \mathrm{sec}, 59 \mathrm{oC} 30 \mathrm{sec} \\
72 \mathrm{oC} 30 \mathrm{sec}(40 \text { cycles })\end{array}$ & 350 & \\
\hline \multirow[t]{2}{*}{ H. bilis } & $\begin{array}{l}\text { OF-CTATGACGGGTATCCGGC } \\
\text { OR-CTCACGACACGAGCTGAC }\end{array}$ & $\begin{array}{l}98 \mathrm{oC} 10 \mathrm{sec}, 55 \mathrm{oC} 30 \mathrm{sec}, \\
72 \mathrm{oC} 1 \text { minute }(35 \text { cycles })\end{array}$ & 718 & \multirow[t]{2}{*}{$\begin{array}{l}\text { Hamada et al. } \\
\text { (2009) }\end{array}$} \\
\hline & $\begin{array}{l}\text { IF- CAGAACTGCATTTGAAACTAC } \\
\text { IR-AAGCTCTGGCAAGCCAGC }\end{array}$ & $\begin{array}{l}98 \mathrm{oC} 15 \mathrm{sec}, 57 \mathrm{oC} 30 \mathrm{sec} \\
72 \mathrm{oC} 30 \mathrm{sec}(35 \mathrm{cycles})\end{array}$ & 418 & \\
\hline \multirow[t]{2}{*}{ H. hepaticus } & $\begin{array}{l}\text { OF-CTATGACGGGTATCCGGC } \\
\text { OR-CTCACGACACGAGCTGAC }\end{array}$ & $\begin{array}{l}98 \mathrm{oC} 10 \mathrm{sec}, 55 \mathrm{oC} 30 \mathrm{sec} \\
72 \mathrm{oC} 1 \text { minute }(35 \mathrm{cycles})\end{array}$ & 718 & \multirow[t]{2}{*}{$\begin{array}{l}\text { Hamada et al. } \\
(2009)\end{array}$} \\
\hline & $\begin{array}{l}\text { IF -GAAACTGTTACTCTG } \\
\text { IR-TCAAGCTCCCCGAAGGG }\end{array}$ & $\begin{array}{l}98 \mathrm{oC} 15 \mathrm{sec}, 56 \mathrm{oC} 30 \mathrm{sec}, \\
72 \mathrm{oC} 30 \mathrm{sec}(35 \mathrm{cycles})\end{array}$ & 405 & \\
\hline
\end{tabular}


Quantitative real time PCR

H. pylori was quantified by real time PCR in all stool DNA samples and worms from the five groups of hamsters, as described (McDaniels et al., 2005; Linke et al., 2010), with minor modifications. The H.pylori ure $A$ gene subunit ureA was targeted for real-time PCR, using primers HpyF1: GGGTATTGAAGCGATGTTTCCT and HpyR1: GCTTTTTTGC-CTTCGTTGATAGT. The reaction mixture included $10 \mu 1 \mathrm{SYBR}$ Green Master Mix (Thermo Scientific, USA), $1.0 \mu 1$ template DNA; $0.5 \mu 1$ of each primer $(0.625 \mu \mathrm{M})$ and $9 \mu 1$ nuclease-free water. Thermocycling conditions using Roche's Light Cycler 1.5 were: $9 \mathrm{~min}$ initial denaturation at $95^{\circ} \mathrm{C}, 40$ cycles of $15 \mathrm{~s}$ at $95^{\circ} \mathrm{C}$ and $60 \mathrm{~s}$ at $60^{\circ} \mathrm{C}$ for the annealing and elongation steps. To quantify number of bacterial cells in samples, a standard curve derived was established using 10-fold serial dilutions of H.pylori DNA from $10^{8}$ cells/ ml. Cell numbers of $H$. pylori cell count in one gram of colorectal feces was ascertained by the standard curve at crossing point and log concentration, as described (Linke et al., 2010). The cell concentration/amount of DNA was calculated to obtain the same initial amount for all templates. Escherichia coli was used as negative control.

\section{In situ localization of $H$. pylori in O. viverrini flukes}

Formalin-fixed and paraffin-embedded tissue sections of $O$. viverrini infected hamster livers were histochemically stained using the Warthin-Starry method (Hartman and Owens, 2012). Briefly, sections were deparaffinized, hydrated to water, rinsed in acidic distilled water $\mathrm{pH}$ 3.8-4.4, and stained with $1 \%$ silver nitrate solution for 45 seconds in a microwave oven. After cooling to room temperature, the slides were immersed in $0.15 \%$ hydroquinone, $5 \%$ gelatin, $2 \%$ silver nitrate (reducing solution) for one minute, rinsed thoroughly in tap water, then dehydrated, cleared and mounted.

Specific localization of $H$. pylori was also undertaken using an immunohistochemistry approach. In brief, hydrated thin tissue sections were subjected to antigen retrieval in $0.01 \mathrm{~mol} / 1$ citrate buffer using a high temperature pressure cooker; endogenous peroxidase activity was blocked with $3 \%$ hydrogen peroxide in methanol. Non-specific staining was minimized by incubation in 5\% normal horse serum in PBS. Sections were probed with anti $H$.pylori rabbit polyclonal antibody (Dako) overnight (primary antibody). After washing with PBS, sections were incubated in biotinylated goat antirabbit IgG-HRP (Dako) for $60 \mathrm{~min}$ (secondary antibody), washed and developed with 3,3'- diaminobenzidine (DAB) (Sigma). Subsequently, sections were counterstained in Mayer's heamatoxylin, cleared, and mounted. Paraffinembedded gastric tissue from $H$. pylori-infected patient was used as positive control. Negative control sections were probed only with secondary antibody.

\section{Statistical analyses}

Chi-square $\left(\mathrm{X}^{2}\right)$ or Fisher's exact test was used to compare the categorical data among treatment groups. One-way ANOVA (Post Hoc test) was used to compare the means of total cell counts per gram feces. $p$ values $\leq 0.05$ were considered to be statistically significant.

\section{Results}

Prevalence of Helicobacter in hamster feces

Figure 1 presents the prevalence of infection with species of Helicobacter as determined by fecal PCRs targeting the 16S rRNA, ureA, H. bilis, and H. hepaticusspecific genes in $O$. viverrini-infected hamster with and without treatments and controls. Baseline prevalence of species of Helicobacter was significantly higher in hamsters with $O$. viverrini infection $(90 \%)$ than in non- $O$. viverrini infected hamsters $(43.3 \%)(\mathrm{p} \leq 0.001)$. Similarly, the prevalence of species of Helicobacter in the $O$. viverrini infected group was significantly higher than both the $O$. viverrini-infected group treated with $\mathrm{ABx}(46.7 \%)$ and the $O$. viverrini-infected group treated with $\mathrm{ABx}$ and

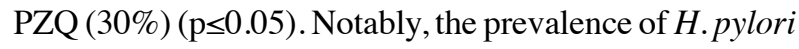
infection (ureA gene positive) was significantly higher in hamsters without $O$. viverrini infection $(33.3 \%)$ than that in non- $O$. viverrini infected hamsters treated with $\mathrm{ABx}$ $(16.7 \%)(\mathrm{p} \leq 0.001)$.

Moreover, the prevalence of $H$. pylori in hamster infected with $O$. viverrini $(73.3 \%)$ was significantly higher than in hamsters infected with $O$. viverrini and treated with $\mathrm{ABx}(36.7 \%)$ and in those treated with both $\mathrm{ABx}$ and PZQ $(16.7 \%)(\mathrm{p} \leq 0.001)$. Likewise, the prevalence of $H$. bilis but not $H$. hepaticus infection was higher in the $O$. viverrini infected hamsters (40\%) than the non-O. viverrini infected rodents $(26.7 \%)(\mathrm{p} \leq 0.001)$. In addition, H. bilis infection was more prevalent in hamsters with $O$. viverrini infection $(26.7 \%)$ than in hamsters with $O$. viverrini treated with $\mathrm{ABx}(13.3 \%)$ and the $O$. viverrini infected group treated with ABx and PZQ (13.3\%) ( $\mathrm{p} \leq 0.05)$. Last, and notable, the prevalence of $H$. pylori and $H$. bilis was lower in hamsters infected with $O$. viverrini and treated with $\mathrm{ABx}$ and $\mathrm{PZQ}$ than in $O$. viverrini-infected hamsters treated only with $\mathrm{ABx}$, and was similar to that in the non-O. viverrini infected hamsters.

\section{Prevalence of Helicobacter pylori in hamster organs}

The prevalence of $H$. pylori in diverse hamster organsstomach, liver, colon, gallbladder including feces from

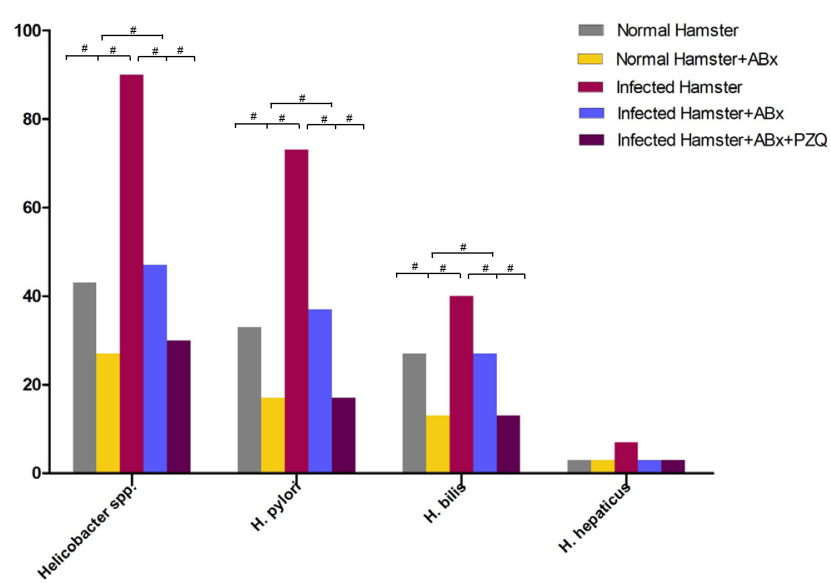

Figure 1. Prevalence of Helicobacter spp. Infection in feces from 5 groups of hamsters. X-axis represents PCR positivity (\%) of Helicobacter 16SrRNA, UreA, H. bilis, and $H$. hepaticus among groups. "Significant difference ( $\mathrm{p} \leq 0.001)$ 


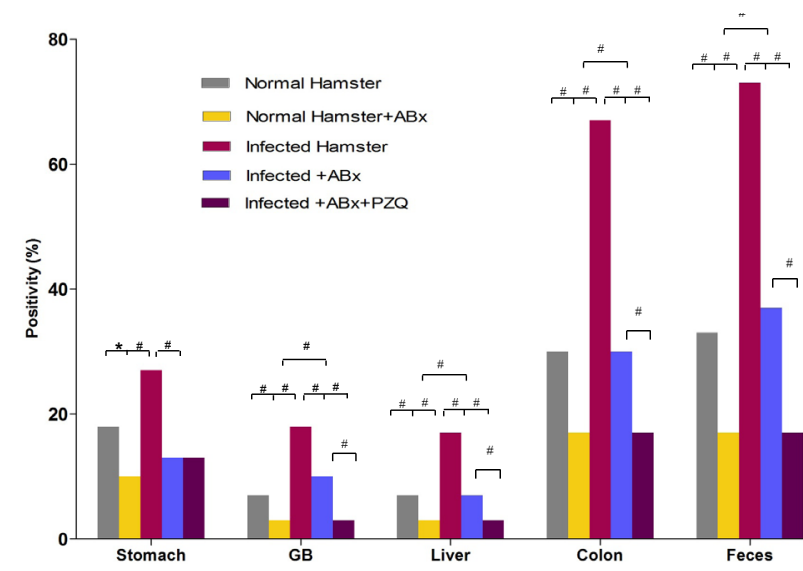

Figure 2. Prevalence of Helicobacter pylori Infection in Various Hamster Organs by PCR. Data (\%) for $\mathrm{H}$. pylori ${ }^{\#}(\mathrm{p} \leq 0.001)$ and $*(\mathrm{p} \leq 0.05)$

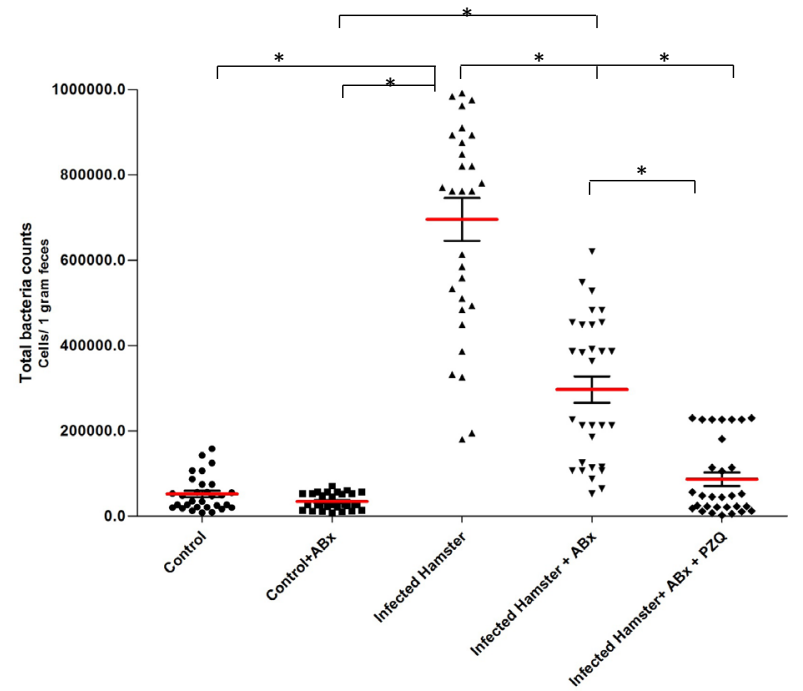

Figure 3. Quantification of Helicobacter pylori in Colorectal Feces of Hamsters Using Quantitative RTPCR. Total bacterial cell counts among the following 5 groups of hamsters are compared; 1. Control (Group 1), 2. Control+ ABx (Group 2), 3. Infected hamster (Group 3), 4. Infected hamster + ABx (Group 4), and 5. Infected hamster $+\mathrm{ABx}+\mathrm{PZQ}($ Group 5). *Significant difference among groups $(\mathrm{p} \leq 0.001)$

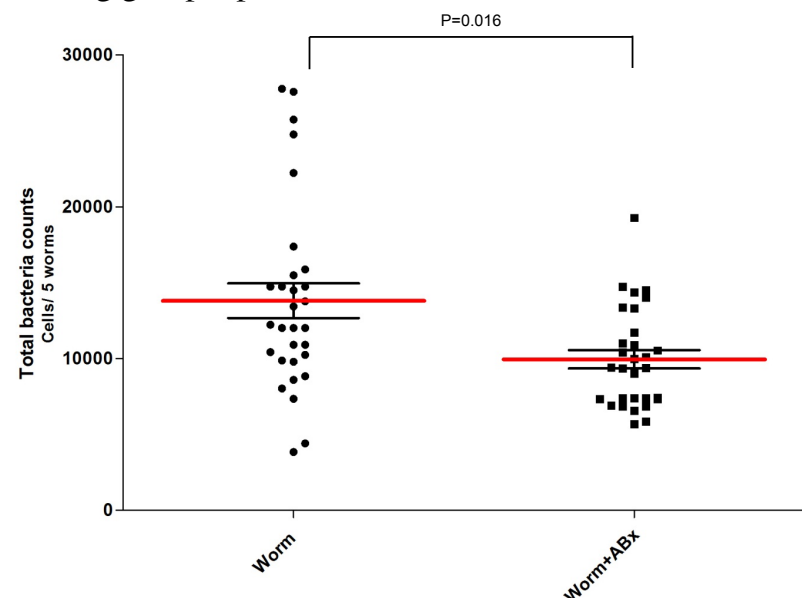

Figure 4. Quantification of Helicobacter pylori in $\boldsymbol{O}$. viverrini from Infected Hamsters (Group 2) and $O$. viverrini from Infected Hamsters Treated with $\mathbf{A B x}$ (Groupp 3). Data are representative of bacterial counts from 5 worms/hamster recovered from each group colon, varied among the treatment groups (Figure 2). $H$. pylori was more common $(>70 \%)$ in colon and feces of $O$. viverrini-infected compared to $<40 \%$ in control hamsters not infected with $O$. viverrini $(\mathrm{p} \leq 0.001)$. After treatment with $\mathrm{ABx}$, the prevalence of $H$. pylori in both colon and colorectal feces declined significantly. The prevalence of $H$. pylori was significantly lower in $O$. viverrini-infected hamsters treated with $\mathrm{ABx}$ and $\mathrm{PZQ}-16.7 \%$ vs $66.7 \%$ and $16.6 \%$ vs. $73.9 \%$ for the colon tissue and feces, respectively ( $\mathrm{p} \leq 0.001)$, levels about half those from the $\mathrm{ABx}$ treated hamsters.

For the liver and gallbladder, in like fashion, significantly higher prevalence of $H$. pylori was seen in hamsters infected with $O$. viverrini compared to non-infected controls $(16.6 \%$ vs $6.7 \%$ and $18.0 \%$ vs $6.7 \%$, respectively). Treatments with $\mathrm{ABx}$ and PZQ of $O$. viverrini-infected hamsters significantly reduced the prevalence of $H$. pylori to less than half of their $\mathrm{ABx}$ baselines in both the liver and gallbladder $(6.7 \%$ vs. $3.3 \%$ and $10.0 \%$ vs $3.3 \%$, respectively). Similar to other organs, infection prevalence of $H$. pylori in the stomach was significantly higher in in O. viverrini infected $(26.7 \%)$ compared to uninfected hamsters $(18.0 \%)(\mathrm{p} \leq 0.05)$. However, the prevalence of $H$. pylori in $O$. viverriniinfected hamsters treated with $\mathrm{ABX}$ and PZQ $(13.3 \%)$ was similar to those treated with $\mathrm{ABx}$ alone $(13.3 \%)$, unlike those organs/ tissues reported above. In overview, the prevalence of $H$. pylori in organs of the $\mathrm{ABx}$ and $\mathrm{PZQ}$ treated hamsters declined to about their $\mathrm{ABx}$-treated baselines.

Quantification H. pylori by quantitative real-time PCR (qPCR)

Levels of H.pylori (H. pylori cell counts) in the five groups of hamsters, as established from the qRT-PCR standard curve, are presented in Figure 3. Significantly divergent intensities of infection with $H$. pylori were

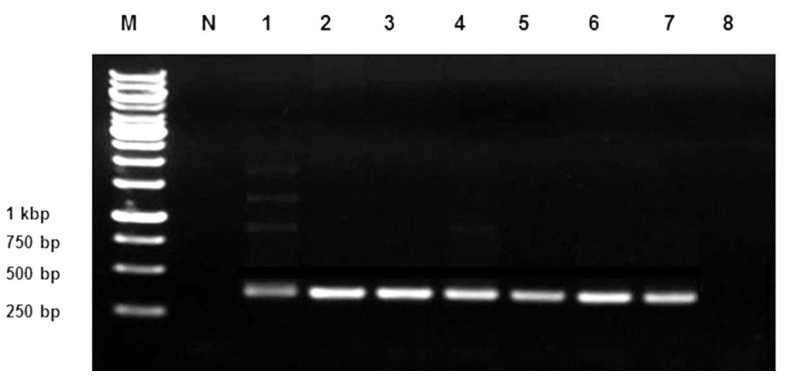

Figure 5. Representative PCR Products Amplified by using Helicobacter pylori Specific Primer (Product Size $=350 \mathrm{bp})$ from $O$. viverrini Worms (Recovered from Group 2) Cultured with Antibiotics upto 4 Weeks, Excretory-secretory (ES) Products, Fresh Worms and $\mathrm{Ov}$ metacercariae. $\mathrm{M}=$ Maker, $\mathrm{N}=$ Negative control, Lane 1=Positive control (H. pylori), Lane 2=Fresh Ov, Lane 3=Ov cultured 1 week, Lane 4=Ov cultured 2 weeks, Lane $5=$ Ov cultured 3 weeks Lane $6=$ Ov cultured 4 weeks, Lane7=ES products and Lane 8=Metacercariae, respectively. Data are reprehensive of PCR products amplified from DNA extracted from 10 worms per duration or DNA from 500 O. viverrini metacercariae. $\mathrm{Ov}=$ Opisthorchis viverrini 


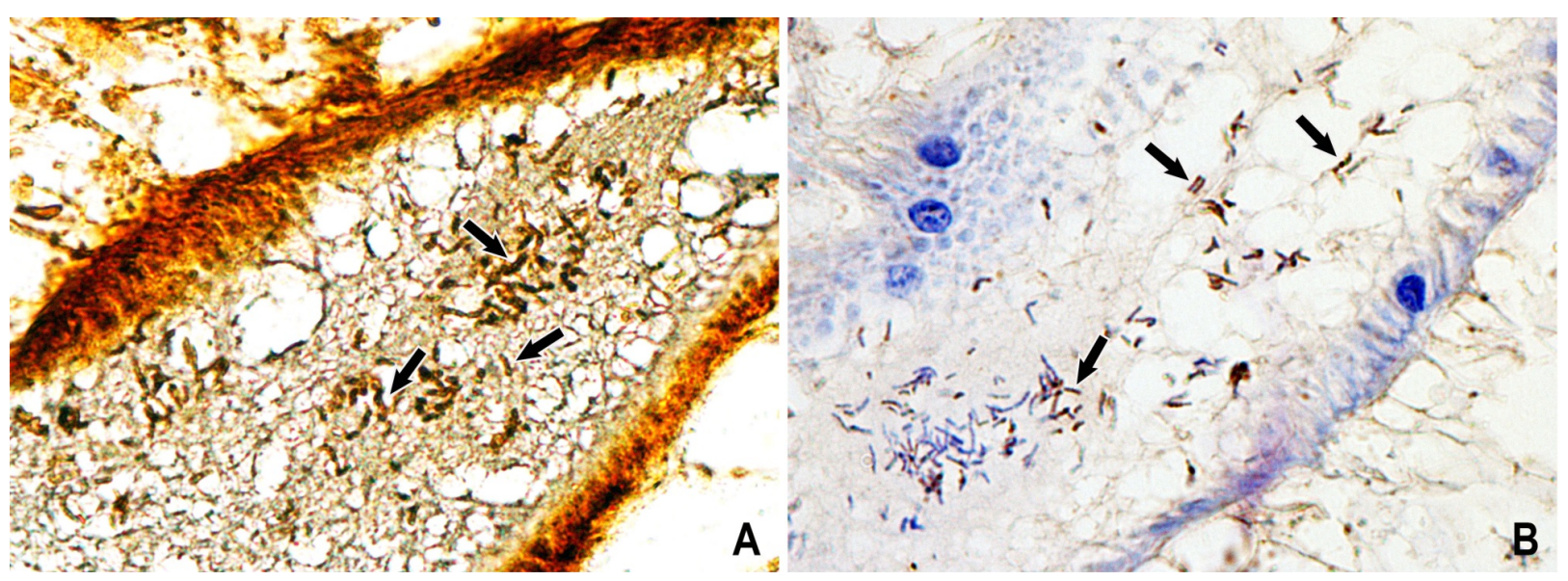

Figure 6. Identification of Helicobacter pylori in the $\boldsymbol{O}$. viverrini Gut. (A) Cluster of $H$. pylori-like (short arrow) stained with the Warthin-Starry method shown as dark brown curved rod-like bacteria in the lumen. (B) Specific detection of $H$.pylori with anti-H. pylori antibody immunostained as a dark brown color (short arrow). Original magnification, $\mathrm{x} 400$

evident: $O$. viverrini-infected hamsters, 695,712 $\pm 270,962$ cells (mean $\pm \mathrm{SD} /$ five worms); O. viverrini infected hamsters treated with ABx, 297,033 $\pm 167,158 ; O$. viverrini infected hamsters treated with $\mathrm{ABX}$ and $\mathrm{PZQ}$, $86,763 \pm 86,585$; uninfected hamsters, 52,105 $\pm 40,004$; and uninfected hamsters treated with $\mathrm{ABx}, 34,497 \pm 18,360$ ( $\leq \leq 0.001)$. Overall, H. pylori infection intensity in $O$. viverrini infected hamsters was significantly greater than that of uninfected hamsters $(\mathrm{p} \leq 0.001)$. Of note, $O$. viverrini infected hamsters treated with $\mathrm{ABx}$ and PZQ showed significant reduction of $H$. pylori infection intensity $(\mathrm{p} \leq 0.001)$, similar to levels detected in the non-O. viverrini-infected hamsters treated or not treated with $\mathrm{ABx}$.

\section{The quantification of $H$. pylori in $O$. viverrini worms}

The $H$. pylori infection intensity in $O$. viverrini recovered from hamsters (mean $=13,806 \pm 6,162$ cells $/ 5$ worms) was significantly greater than that in $O$. viverrini from infected hamsters treated with $\mathrm{ABx}(9,951 \pm 3,220)$ (Figure 4).

\section{Detection of H. pylori in cultured O. viverrini worms}

To investigate the presence of $H$. pylori in longterm culture with $\mathrm{ABx}$, we did examine by PCR up to 4 weeks culture as well as in fresh worms, ES products and metacercariae. The results showed that $H$. pylori could be observed in fresh recovered adults, all cultured worms, and ES products but not in the metacercariae of O. viverrini (Figure 5).

\section{In situ localization of $H$. pylori in O. viverrini}

To directly investigate the existence/presence of $H$. pylori in the $O$. viverrini flukes, in situ localization using the Warthin-Starry technique and immunohistochemistry was undertaken. Warthin-Starry staining revealed dark brown curved rod-like structures that clearly resembled H. pylori within the lumen of the gut of the flukes (Figure 6A). In like fashion, immunohistochemical staining with antibody specific for $H$. pylori showed strong, positive signals for the bacteria in the lumen of the gut of the flukes (Figure 6B).

\section{Discussion}

Cholangiocarcinoma is highly prevalent in Asian countries; particularly in Thailand (Shin et al., 2010) and, besides dietary carcinogens, the main risk factor is infection with the human liver flukes, O. viverrini and Clonorchis sinensis (Sripa et al., 2007; Sripa and Pairojkul, 2008; Petney et al., 2013; Sithithaworn et al., 2014). Other risk factors associated with the infection such as microbiota in the biliary system may also be involved (Plieskatt et al., 2013). We recently found a significant association between $H$. pylori or mixed $H$. pylori and $H$. bilis but not $H$. hepaticus infection and CCA in patients from Northeast Thailand, a region highly endemic for opisthorchiasis (Boonyanugomol et al., 2012b; 2012c). However, which species of Helicobacter associate with opisthorchiasis, and why, remain unclear. We show here, for the first time in a rodent model a human opisthorchiasis, significantly higher prevalence of co-infection with $H$. pylori and $H$. bilis but not $H$. hepaticus in $O$. viverriniinfected and uninfected hamsters. Interestingly, clearance of the liver fluke infection following treatment with PZQ significantly reduced the prevalence and intensity of $H$. pylori in $O$. viverrini-infected hamsters suggested a strong inter-relationship between the two pathogens. In addition, in situ localization revealed H. pylori in the gut of the worm and the presence of of $H$.pylori in worms cultured with lengthy periods in antibiotics supports that adult $O$. viverrini may act as a reservoir for $H$. pylori.

Species of Helicobacter including H. pylori, $H$. hepaticus and $H$. bilis have been described from several other mammals as well as from people (Fox et al., 1995; Pellicano et al., 2008; Goldman et al., 2010). Fox et al. (2009) reported hamsters naturally infected with $H$. bilis and that aged animals showed chronic hepatitis, hepatic dysplasia, fibrosis, and biliary hyperplasia. During other liver fluke infections, $H$. bilis-like bacteria has been identified in the intrahepatic bile ducts of rats experimentally infected with Fasciola hepatica (Foster, 1984). Our study reported a significant higher prevalence of $H$. pylori and $H$. bilis but not $H$. hepaticus in the colon feces of $O$. viverrini infected hamsters 
compared to uninfected hamsters both before and after $\mathrm{ABx}$ treatment. We further investigated the source of the higher fecal infection by examining the prevalence of $H$. pylori in the stomach, liver, gallbladder, and colon mucosa. Helicobacter spp. have been observed previously in these sites (Fox et al., 1995). The findings presented here demonstrated infection of liver and gallbladder with $H$. pylori, and indicated that the H.pylori in these organs originated from the liver fluke in the bile ducts rather than from microbiome of the hamster stomach. The findings indicated that infection with $O$. viverrini influenced the higher positivity and intensity in the feces since levels of $H$. pylori significantly declined following treatments with ABx and PZQ (Figures 2 and Figure 3). The source of Helicobacter infection of the liver and biliary system may be ascending infection through the small intestine (Pellicano et al., 2008). Partial bile duct obstruction by the flukes as well as host inflammatory responses cause bile stasis and facilitate ascending infection and cholangitis (Carpenter, 1998; Sripa, 2003). We recently reported higher number of bacterial species in $O$. viverrini infected hamster bile and also colorectal feces (Plieskatt et al., 2013). Although Helicobacter is bile-sensitive, the mechanism by which colonization by this microbe of the biliary system is accomplished is uncertain. It has been suggested that the 'acidification' of bile through reflux into the low $\mathrm{pH}$ of the stomach precipitates inhibitory bile acids, thereby allowing colonization by H.pylori (Hynes et al., 2003; Shao et al., 2008). In case of opisthorchiasis, the infection by the flukes can disturb bile acid composition (Wongpaitoon et al., 1988; Wonkchalee et al., 2012) that may reduce alkalinity of the bile, leading to damage to biliary epithelia and inflammation (Strazzabosco et al. 2000). All these phenomena may facilitate conditions that favor colonization by Helicobacter during concurrent infection with liver flukes.

Whether the presence of $H$.pylori in the biliary system during opisthorchiasis occurs only in the bile and biliary mucosa or also within the fluke had until now remained unresolved. Here we demonstrated in situ localization of H. pylori histochemically and immunohistochemically in the gut or gastrodermal of the fluke. Accidental contamination during specimen preparation cannot account for these new findings since $H$. pylori was qualitatively and quantitatively detected in the thoroughly washed worms and cultured in the presence of $\mathrm{ABx}$ for more than a month. However, the origin of infection of the liver fluke with Helicobacter spp. is unclear. The fluke likely ingests bile contents and biliary epithelium for nutrition (Sripa, 2003; Sripa et al., 2007), and H. pylori is likely ingested in the bile by the fluke. We postulate that $H$. pylori can colonize and propagate in the gut epithelium of $O$. viverrini and, in turn, continue to be released back into bile with the excretions and secretions (ES) of the flukes. DNA from H. pylori can be detected by PCR in the ES products of the cultured worms (Figure 5). The epithelia of the gut of $O$. viverrini may provide conditions favorable to Helicobacter i.e. acidic to neutral $\mathrm{pH}$, mucus as well as specific adhesion molecules (Sachs et al, 2011; Dunne et al., 2014). Together, these new findings suggest that $O$. viverrin $i$ within the mammalian hepatobiliary system may be a reservoir of $H$. pylori.

The elevated prevalence of Helicobacter spp. in $O$. viverrini-infected hamsters raises the question of whether infection with Helicobacter contributes to pathogenesis of hepatobiliary diseases as well as CCA. Hepatobiliary diseases caused by species of Helicobacter in naturally infected hamsters (Fox et al., 2009; Boonyanugomol et al. 2012b; 2012c) are similar to those of $O$. viverrini (Lvova et al., 2012; Mairiang et al., 2012). Indeed, the chronic lesions ascribed to liver fluke infection in both hamsters and humans, i.e., cholangitis, biliary hyperplasia and metaplasia, and periductal fibrosis or even CCA may be due, in part, to Helicobacter-associated hepatobiliary disease. $H$. pylori and $H$. bilis DNA has been isolated from liver tissue of humans with CCA and cholecystitis/ cholelithiasis from Thai patients living in regions endemic for opisthorchiasis (Boonyanugomol et al. 2012b; 2012c). Moreover, serological assays indicate active infection with $H$. pylori and $H$. bilis in Thais at high risk for CCA(Pisani et al., 2008). H. bilis was also associated with biliary cancers in two high-risk populations in Japan and Thailand (Matsukura et al., 2002). The strong association between liver fluke and H. pylori or H. bilis infection reported in this study supports a role of these Helicobacter species in the hepatobiliary diseases known during opisthorchiasis.

In conclusion, this study in a well-controlled hamster model of infection revealed a significant relationship between $O$. viverrini and Helicobacter spp., specifically $H$. pylori and H. bilis. The in situ localization of $H$. pylori in the gut of the fluke supports the hypothesis that $O$. viverrini may be a reservoir of this carcinogenic bacterium. The co-infection may orchestrate in the pathogenesis of liver fluke-induced hepatobiliary diseases including CCA.

\section{Acknowledgements}

This study was partially supported by the Commission on Higher Education, Thailand, under the Program Strategic Scholarships for Frontier Research Network for the Joint PhD Program Thai Doctoral Degree, Faculty of Medicine, Khon Kaen University, Thailand (Grant No. I56110), the National Health Security Office of Thailand (NHSO), the Thailand Research Fund (TRF), and the Tropical Medicine Research Center from the National Institute of Allergy and Infectious Diseases (NIAID), National Institutes of Health (NIH) award number P50AI098639 (PI, Sripa, B.). BS is a Senior TRF Scholar (2013-2016). The content of this report is solely the responsibility of the authors and does not necessarily represent the official views of the NIH and other funders.

\section{References}

Boonyanugomol W, Chomvarin C, Baik SC, et al (2011). Role of cagA-positive Helicobacter pylori on cell proliferation, apoptosis, and inflammation in biliary cells. Dig Dis Sci, 56, 1682-92.

Boonyanugomol W, Chomvarin C, Song JY, et al (2012a). Effects of Helicobacter pylori gamma-glutamyltranspeptidase on apoptosis and inflammation in human biliary cells. Dig Dis Sci, 57, 2615-24.

Boonyanugomol W, Chomvarin C, Sripa B, et al (2012b). 
Helicobacter pylori in Thai patients with cholangiocarcinoma and its association with biliary inflammation and proliferation. HPB (Oxford), 14, 177-84.

Boonyanugomol W, Chomvarin C, Sripa B, et al (2012c). Molecular analysis of Helicobacter pylori virulentassociated genes in hepatobiliary patients. HPB (Oxford), 14, 754-63.

Bouvard V, Baan R, Straif K, et al (2009). A review of human carcinogens--Part B: biological agents. Lancet Oncol, 10, $321-2$.

Carpenter, HA (1998). Bacterial and parasitic cholangitis. Mayo Clin Proc, 73, 473-8.

Cid TP, Fernandez MC, Benito Martinez S, et al (2013). Pathogenesis of Helicobacter pylori infection. Helicobacter, 18, $12-7$.

Dunne C, Dolan B, Clyne M (2014). Factors that mediate colonization of the human stomach by Helicobacter pylori. World J Gastroenterol, 20, 5610-24.

Foster, JR (1984). Bacterial infection of the common bile duct in chronic fascioliasis in the rat. J Comp Pathol, 94, 175-81.

Fox JG, Shen Z, Muthupalani S (2009). Chronic hepatitis, hepatic dysplasia, fibrosis, and biliary hyperplasia in hamsters naturally infected with a novel Helicobacter classified in the H. bilis cluster. J Clin Microbiol, 47, 3673-81.

Fox JG, Yan LL, Dewhirst FE, et al (1995). Helicobacter bilis sp. nov, a novel Helicobacter isolated from bile, livers, and intestines of aged, inbred mouse strains. J Clin Microbiol, 33, 445-54.

Goldman CG, Mitchell HM (2010). Helicobacter spp. other than Helicobacter pylori. Helicobacter, 15, 69-75.

Hartman DJ, Owens SR (2012). Are routine ancillary stains required to diagnose Helicobacter infection in gastric biopsy specimens? An institutional quality assurance review. Am J Clin Pathol, 137, 255-60.

Hatakeyama M (2014). Helicobacter pylori CagA and gastric cancer: a paradigm for hit-and-run carcinogenesis. Cell Host Microbe, 15, 306-16.

Huai JP, Ding J, Ye XH, et al (2014). Inflammatory bowel disease and risk of cholangiocarcinoma: evidence from a meta-analysis of population-based studies. Asian Pac J Cancer Prev, 15, 3477-82.

Hynes SO, McGuire J, Falt T, et al (2003). The rapid detection of low molecular mass proteins differentially expressed under biological stress for four Helicobacter spp. using ProteinChip technology. Proteomics, 3, 273-8.

IARC (2012). Biological agents. Vo. 100 B. a review of human carcinogens. IARC Monogr Eval Carcinog Risks Hum, 100, 1-441.

Khoshnegah J, Jamshidi S, Mohammadi M, et al (2011). The efficacy and safety of long-term Helicobacter species quadruple therapy in asymptomatic cats with naturally acquired infection. J Feline Med Surg, 13, 88-93.

Linke S, Lenz J, Gemein S, et al (2010). Detection of Helicobacter pylori in biofilms by real-time PCR. Int J Hyg Environ Health, 213, 176-82.

Lvova MN, Tangkawattana S, Balthaisong S, et al (2012). Comparative histopathology of Opisthorchis felineus and Opisthorchis viverrini in a hamster model: an implication of high pathogenicity of the European liver fluke. Parasitol Int, 61, 167-72.

Mairiang E, Laha T, Bethony JM, et al (2012). Ultrasonography assessment of hepatobiliary abnormalities in 3359 subjects with Opisthorchis viverrini infection in endemic areas of Thailand. Parasitol Int, 61, 208-11.

Malhi H, Gores GJ (2006). Cholangiocarcinoma: modern advances in understanding a deadly old disease. J Hepatol, 45, 856-67.
Mateos-Munoz B, Perez-de-la-Serna J, Ruiz-de-Leon A, et al (2013). Enterohepatic Helicobacter other than Helicobacter pylori. Rev Esp Enferm Dig, 105, 477-84.

Matsukura N, YokomuroS, Yamada S, et al (2002). Association between Helicobacter bilis in bile and biliary tract malignancies: H. bilis in bile from Japanese and Thai patients with benign and malignant diseases in the biliary tract. Jpn J Cancer Res, 93, 842-7.

Matsumoto K, Onoyama T, Kawata S, et al (2014). Hepatitis B and $\mathrm{C}$ virus infection is a risk factor for the development of cholangiocarcinoma. Intern Med, 53, 651-4.

McDaniels AE, Wymer L, Rankin C, et al (2005). Evaluation of quantitative real time PCR for the measurement of Helicobacter pylori at low concentrations in drinking water. Water Res, 39, 4808-16.

Murphy G, Michel A, Taylor PR, et al (2014). Association of seropositivity to Helicobacter species and biliary tract cancer in the ATBC study. Hepatology, 60, 1963-71.

Pellicano R, Mazzaferro V, Grigioni WF, et al (2004). Helicobacter species sequences in liver samples from patients with and without hepatocellular carcinoma. World J Gastroenterol, 10, 598-601.

Pellicano R, Ménard A, Rizzetto M, et al (2008). Helicobacter species and liver diseases: association or causation? Lancet Infect Dis, 8, 254-60.

Petney TN, Andrews RH, Saijuntha W, et al (2013). The zoonotic, fish-borne liver flukes Clonorchis sinensis, Opisthorchis felineus and Opisthorchis viverrini. Int $J$ Parasitol, 43, 1031-46.

Pisani P, Whary MT, Nilsson I, et al (2008). Cross-reactivity between immune responses to Helicobacter bilis and Helicobacter pylori in a population in Thailand at high risk of developing cholangiocarcinoma. Clin Vaccine Immunol, 15, 1363-8.

Plieskatt JL, Deenonpoe R, Mulvenna JP, et al (2013). Infection with the carcinogenic liver fluke Opisthorchis viverrini modifies intestinal and biliary microbiome. FASEB J, 27, 4572-84.

Rizvi S, Gores GJ (2013). Pathogenesis, diagnosis, and management of cholangiocarcinoma. Gastroenterology, 145, 1215-29.

Sachs G, Scott DR, Wen Y, (2011). Gastric infection by Helicobacter pylori. Curr Gastroenterol Rep, 13, 540-6.

Shao C, Zhang Q, Sun Y, et al (2008). Helicobacter pylori protein response to human bile stress. J Med Microbiol, 57, 151-8.

Shin HR, Oh JK, MasuyerE, et al (2010). Comparison of incidence of intrahepatic and extrahepatic cholangiocarcinoma-focus on East and South-Eastern Asia. Asian Pac J Cancer Prev, 11, 1159-66.

Sithithaworn P, Yongvanit P, Duenngai K, et al (2014). Roles of liver fluke infection as risk factor for cholangiocarcinoma. $J$ Hepatobiliary Pancreat Sci, 21, 301-8.

Sripa B (2003). Pathobiology of opisthorchiasis: an update. Acta Trop, 88, 209-20.

Sripa B, Brindley PJ, Mulvenna J, et al (2012). The tumorigenic liver fluke Opisthorchis viverrini-multiple pathways to cancer. Trends Parasitol, 28, 395-407.

Sripa B, Kaewkes S, Sithithaworn P, et al (2007). Liver fluke induces cholangiocarcinoma. PLoS Med, 4, 201.

Sripa B, Pairojkul C (2008). Cholangiocarcinoma: lessons from Thailand. Cur Opin Gastroenterol, 24, 349-56.

Strazzabosco M, Spirli C, Okolicsanyi L, (2010). Pathophysiology of the intrahepatic biliary epithelium. J Gastroenterol Hepatol, 15, 244-53.

Wongpaitoon V, Thuvasethakul P, Kurathong S, et al (1988). Biliary bile acids and lipids composition in Opisthorchiasis viverrini. J Med Assoc Thai, 71, 394-9. 
Wonkchalee O, Boonmars T, Kaewkes S, et al (2012). Comparative studies on animal models for Opisthorchis viverrini infection: host interaction through susceptibility and pathology. Parasitol Res, 110, 1213-23.

Wu Q, He XD, Yu L, et al (2012). The metabolic syndrome and risk factors for biliary tract cancer: a case-control study in China. Asian Pac J Cancer Prev, 13, 1963-9.

Zhou D, Wang JD, Weng MZ, et al (2013). Infections of Helicobacter spp. in the biliary system are associated with biliary tract cancer: a meta-analysis. Eur J Gastroenterol Hepatol, 25, 447-54. 